\title{
Clinical performance of the levonorgestrel intra-uterine system in routine use by the UK Family Planning and Reproductive Health Research Network*: 12-month report
}

\author{
Michael Cox, FRCOG, MFFP, Consultant Obstetrician and Gynaecologist, Sarah Blacksell, BA, Research Fellow \\ Institute of Population Studies, School of Postgraduate Medicine and Health Sciences. University of Exeter, Exeter, UK.
}

Correspondence: Michael Cox, Institute of Population Studies, School of Postgraduate Medicine and Health Sciences. University of Exeter, Hoopern House, 101 Pensylvania Road, Exeter EX4 6TD. Tel: 01392 257936; Fax: 01392490870

(Accepted April 16 $\left.6^{\text {th }}, 2000\right)$

\begin{abstract}
Summary
Doctors working in general practice and at family planning clinics throughout the UK who collaborate in the UK Family Planning and Reproductive Health Research Network were responsible for the fitting of 692 Levonorgestrel Intra-uterine Systems (LNG IUS). This study was undertaken to determine the performance of the LNG IUS in British women in routine clinical use.

The 12 months cumulative life-table event rates were: pregnancy 0.6, expulsion 4.5. The continuation rate was 70.6. Removals were also required for side effects, which may be due to absorbed levonorgestrel, the removal rate being 7.4. Positive effects include effective contraception and considerably reduced quantity of bleeding for most participants.
\end{abstract}

A need was identified to counsel women about the early bleeding problems, including the possibility of oligomenorrhoea or amenorrhoea. This is considered to be very important as it will help women to persevere so that they can enjoy the longer-term benefits.

*Principal investigators: V Annandale (Norwich), A Barnett (Exeter), P Barnes (Richmond, London), J Bateman (Portsmouth), J Bland (Nuneaton), D Booker (Newport), R Bradbury (Barnsley), S Brown (Birmingham), G Cardy (Bristol), S Carr (Glasgow), H Cooling (Bristol), M Cox (Nuneaton), D Craggs (Southampton), J Dewsbury (Birmingham), J Elstub (Richmond, London), S Green (Cumbria), B Hanson (Wiltshire), T Laverty (Wiltshire), A Main (Richmond, London), H Massil (London, CHSL), C Nash (Norwich), E Oloto (Leeds), R Owen (Taunton), S Randall (Portsmouth), S Richardson (W Yorkshire), S Rowlands (Biggleswade), J Rumsey (Birmingham), C Smith (Thetford), J Tattersall (Cumbria), A Taylor (Sheffield), A Thomas (Newport), R Tolcher (Southampton), C Tupper (Cumbria), A Turner (Wiltshire), M Walling (Boston), C Watson (London, CHSL), EM Watt (Bristol).

\section{Key words}

intra-uterine contraception, levonorgestrel intra-uterine system; 12-month findings

\section{Key message points}

At 12 months of use the LNG IUS (Mirena ${ }^{\circledR}$ ) has been shown to have a very low pregnancy rate and a low expulsion rate.

- The rate of removal for complaints of bleeding or bleeding with pain was high.

- There is a need for careful counselling before fitting to prepare women for changes in bleeding patterns during the first 12 months of use.

- Removals were also required for side effects which may be due to absorbed levonorgestrel.

- The reduction in duration and quantity of periods in many women were significant advantages.

\section{Introduction}

The concept of intra-uterine administration of a progestinonly contraceptive as an alternative to oral, parenteral or subdermal implants was first considered in $1970{ }^{1}$ Experiments with various slow-release membranes and intra-uterine contraceptive device (IUD)-carrying frames resulted in the development of an intra-uterine system (IUS) releasing levonorgestrel, now marketed as Mirena ${ }^{\circledR}$. It was introduced in Finland in 1990 and in the UK in May 1995.

Very low pregnancy rates have been reported; between zero and 0.6 cumulative gross rates per 100 users at 12 months (Table 1). Very low pregnancy rates have also been reported after up to 7 years of use with a system containing $60 \mathrm{mg}$ of levonorgestrel. ${ }^{5,6}$ Expulsion rates have been similar to that of other IUDs. For example the $\mathrm{WHO}^{7}$ reported a rate of 6.4 per 100 women at 12 months. The Mirena ${ }^{\circledR}$ is recommended for use up to 5 years.

The levonorgestrel suppresses the endometrium, which remains in an atrophic state as long as the LNG IUS remains in situ ${ }^{8}$ and therefore changes in menstrual patterns are likely to occur. Sivin and Stern ${ }^{9}$, reporting a multicentre study, commented that 'menstrual patterns during contraception were radically altered by the LNG IUS'.

Several randomised trials have found that discontinuation rates because of heavy or prolonged menstrual blood flow were significantly lower with the LNG IUS than with copper IUDs. ${ }^{10}$ Sivin et al ${ }^{11}$ found that discontinuation attributable to amenorrhoea was the principal contributor to differences in continuation rates between the LNG IUS and the Copper T 380 Ag IUD.

There is a need to determine the performance of the LNG IUS in British women, with particular regard to the nature and incidence of side effects.

\section{Method}

The study is an ongoing, open, single group, multicentre phase III study in out-patients. Doctors working in general practice and at family planning clinics throughout the UK who co-operate in the UK Family Planning and Reproductive Health Research Network were responsible for the LNG IUS fittings reported in this paper.

The study is sponsored by Leiras Oy and Schering Health Care Limited. Ethics Committee approval for the study was obtained at all centres. The system consists of a T-shaped polyethylene frame carrying a hormone cylinder containing $52 \mathrm{mg}$ of levonorgestrel. The cylinder is covered with a polydimethylsiloxane membrane which regulates the release of $20 \mu \mathrm{g}$ of levonorgestrel per day.

Selection for fitting was according to the doctor's own clinical judgement, in parous women attending for family planning advice and requesting intra-uterine contraception. Before fitting all women were counselled regarding the possible effects of intra-uterine contraception, including the 
Table 1 The LNG IUS: cumulative closure rates at 12 months per 100 users

\begin{tabular}{llrrr}
\hline Study & Country & N & Pregnancy & Pain/bleeding \\
\hline Andersson ${ }^{2} 1994$ & Scandinavia & 1821 & 0.1 & 5.8 \\
Sivin $^{3} 1984$ & Hungary & 1125 & 0.2 & 3.4 (net) \\
ICMR $^{4} 1989$ & India & 475 & 0.0 & 6.0 (gross) \\
Present study 1999 & UK & 692 & 0.6 & 8.9 \\
\hline
\end{tabular}

* combined rate for the two categories of removal for 'bleeding problems' and 'bleeding and pain' (Table 5).

possible effect of levonorgestrel on bleeding and menstruation. Those enrolled were parous women aged 18-45 years, exposed to the risk of pregnancy, in good health and with normal menstrual cycles. They were fitted with their LNG IUS more than 6 weeks after pregnancy ended. After entry to the study, the subjects are being followed for 5 years.

After insertion of the LNG IUS, each subject was requested to return for follow-up examination at 3 months, 12 months and annually thereafter. Baseline data and details of the fitting procedure and follow-up visits were forwarded to the Institute of Population Studies, School of Postgraduate Medicine and Health Sciences, Exeter University, where the data was processed and analysed. Event rates were calculated using the life table method. ${ }^{12}$ Adverse events were classified using the WHO Adverse Reaction Dictionary (version 1/1995). A programme of comprehensive monitoring was introduced in 1996 using EU GCP Guidelines.

At the close of recruitment, 692 women had been recruited who met the inclusion criteria. This paper concentrates on data from this per protocol sample for the first 12 months of use. The age distribution is shown in Table 2.

Table 2 Age distribution

\begin{tabular}{lcc}
\hline Age group & $\mathrm{N}$ & Percent \\
\hline $18-24$ & 67 & 10 \\
$25-29$ & 145 & 21 \\
$30-34$ & 165 & 24 \\
$35-39$ & 195 & 28 \\
$40-45$ & 117 & 17 \\
Total & 689 & 100 \\
\hline
\end{tabular}

3 missing observations

\section{Results}

Demographic characteristics

The age distribution is shown in Table 2. The mean age of the study population was 33 years (range 18-45 years). All the women were parous: $70.4 \%$ had given birth to one or two children and $29.5 \%$ had given birth to three or more.

\section{Contraceptive prior to fitting}

The contraceptive method used prior to fitting is shown in Table 3.

\section{Fitting experiences}

In the majority of cases fitting the LNG IUS presented no difficulties. Investigators reported $11 \%$ of fittings as 'difficult'. No relationship was found between the ease of fitting and parity, status of fitter, interval since pregnancy or breast feeding. There were 20 reported failed fittings. All were in the age range $28-43$ and low parity seemed to be a factor with nine women being para 1 .
Table 3 Contraceptive used prior to LNG IUS fitting

\begin{tabular}{lrr}
\hline Method & N & Percent \\
\hline IUD & 212 & 31 \\
Oral contraception & 135 & 20 \\
Injectable & 14 & 2 \\
Condom & 234 & 34 \\
Cap/diaphragm & 16 & 2 \\
Rhythm/withdrawal & 9 & 1 \\
More than one & 9 & 1 \\
Other & 9 & 1 \\
None & 42 & 6 \\
Unknown & 12 & 2 \\
Total & 692 & 100 \\
\hline
\end{tabular}

Only a minority of fittings $(14 \%)$ needed dilatation. Of these 93 cases, $28(30 \%)$ were described as 'difficult' by the investigator. No insertion difficulty was reported in $89 \%$ of cases. Unexpectedly, insertion difficulties were reported in $4 \%$ of the under- 25 age group, but in $14 \%$ of the $30-34$ year age group. The frequency of pain at insertion is shown in Table 4. Fitting was comfortable for most study subjects, with $78 \%$ experiencing no pain or only mild pain. The numbers offered or given analgesia or local anaesthesia are not known.

Table 4 Pain at insertion

\begin{tabular}{lrr}
\hline & N & Percent \\
\hline Severe & 23 & 3 \\
Moderate & 129 & 19 \\
Mild & 316 & 46 \\
None & 219 & 32 \\
Total & 687 & 100 \\
\hline
\end{tabular}

5 missing observations

Life table closure rates

The cumulative gross rates for closure of study at 3 and 12 months are given in Table 5. These show the LNG IUS to have a low pregnancy and expulsion rate. The continuation rate at 12 months was 70.6 .

Pregnancy

The cumulative gross rate for pregnancy was 0.2 (confidence interval (CI) 0.0-0.9) at 3 months and 0.6 (CI 0.1-1.7) at 12 months (Table 5). Three pregnancies occurred during the first 12 months. Two resulted in spontaneous abortion and one in a missed abortion.

Case report 1. The pregnancy occurred 3 months after fitting. The subject was admitted to hospital with a threatened abortion when the thread could not be seen. She wished to continue with the pregnancy. At a later date an ultrasound scan failed to show the system and the blood levonorgestrel level confirmed its absence. A spontaneous abortion occurred at 21 weeks. The system was probably expelled prior to conception. 
Table 5 Cumulative life-table closure gross rates per 100 users at 3 and 12 months (per protocol sample of 692)

\begin{tabular}{|c|c|c|c|c|c|c|}
\hline & $\mathrm{N}$ & $\begin{array}{l}3 \text { Months } \\
\text { Gross rate }\end{array}$ & CI & $\mathrm{N}$ & $\begin{array}{l}12 \text { Months } \\
\text { Gross rate }\end{array}$ & CI \\
\hline Pregnancy & 1 & 0.2 & $0.0-0.9$ & 3 & 0.6 & $0.1-1.7$ \\
\hline Expulsion & 18 & 2.8 & $1.5-4.1$ & 27 & 4.5 & $2.8-6.1$ \\
\hline Bleeding problems & 15 & 2.4 & $1.2-3.6$ & 43 & 7.6 & $5.4-9.9$ \\
\hline Pain & 7 & 1.1 & $0.4-2.2$ & 12 & 2.1 & $0.9-3.3$ \\
\hline Bleeding and pain & 10 & 1.6 & $0.8-2.9$ & 17 & 2.9 & $1.5-4.2$ \\
\hline Perforation & 0 & 0.0 & $0.0-0.0$ & 0 & 0.0 & $0.0-0.0$ \\
\hline PID & 1 & 0.1 & $0.0-0.8$ & 6 & 1.1 & $0.4-2.4$ \\
\hline Other complaint associated with LNG IUS & 14 & 2.2 & $1.0-3.4$ & 40 & 7.4 & $5.2-9.7$ \\
\hline Planning pregnancy & 0 & 0.0 & $0.0-0.1$ & 15 & 3.1 & $1.5-4.7$ \\
\hline Other reason & 8 & 1.2 & $0.5-2.4$ & 20 & 3.5 & $2.0-5.1$ \\
\hline Medical problem not related to IUS use & 2 & 0.3 & $0.0-1.2$ & 5 & 1.0 & $0.3-2.2$ \\
\hline Total discontinuations & 76 & 11.3 & & 188 & 29.4 & \\
\hline Continuation & & 88.7 & & & 70.6 & \\
\hline
\end{tabular}

Case report 2. Pregnancy occurred at 5 months of use with the LNG IUS in-situ. At 2 months of use, the subject had complained of spotting, amenorrhoea and some cramp-like pains, but was happy with the IUS. She returned at 5 months of use, having had no proper menses since insertion but now feeling pregnant. The IUS was removed easily. The pregnancy ended in spontaneous abortion at 6 weeks.

Case report 3. Pregnancy occurred at 10 months of use. The subject visited the clinic at 6 months of use complaining of irregular and sometimes heavy bleeding, but made no further visits. Information was received through a health visitor that the subject had a missed abortion and evacuation of retained products of conception at 14 weeks of gestation. The LNG IUS was reported to have been found during the procedure.

\section{Removal following a complaint of bleeding or bleeding with pain}

A total of 60 women had the system removed at or before 12 months of use following a complaint of bleeding problems or bleeding and pain together (Table 6). The average length of use before removal of the system for these reasons at 12 months of use was 4.8 months. The type of complaint leading to removal of the system following a complaint of bleeding or bleeding and pain is shown in Table 6 . In more than half $(33 / 60 ; 55 \%)$ of these cases, the subject complained of continuous or persistent vaginal loss. Some women originally in the study had their bleeding problems treated with hormones and then had to be withdrawn from the sample as this contradicted the protocol. Data on other methods of treating bleeding or pain was not collected systematically.

Table 6 Bleeding problems leading to removal at or before 12 months of use

\begin{tabular}{lcc}
\hline Reason for removal & Number & Percent \\
\hline Continuous/persistent vaginal loss & 33 & 55 \\
Irregular bleeding & 11 & 18 \\
Longer periods & 9 & 15 \\
Heavier periods/‘bleeding' & 7 & 12 \\
Total & 60 & 100
\end{tabular}

Other medical complaints requiring removal

The rate for this category of removals was 7.4 at 12 months (Table 5). Within this category, the majority of removals were due to a range of symptoms, some of which may be related to contraceptive steroid use; this is discussed below.
Since in some cases these were described as groups of symptoms, with no one principal reason for removal, these are therefore listed in Table 7 by frequency of occurrence. The complaints of oligo/amenorrhoea were not included in the category 'bleeding problems' of Table 5.

\section{Serious adverse events}

$P I D$. The diagnosis depended on the judgement of the clinician involved. By 12 months, six systems had been removed following a diagnosis of PID, (though the diagnosis was doubtful in two), the removal rate being 1.1 at 12 months (Tables 5 and 8). All were treated successfully. The relationship of PID to the LNG IUS is uncertain and is discussed below.

Deaths and other serious adverse events. Two accidental deaths occurred before 12 months of use, one by drowning and one in a fire. The following cases also occurred:

Case 1: Age 29. Ruptured ovarian cyst 4 months after fitting. Right salpingo-oophorectomy was performed. LNG IUS was not removed. This subject is also the first in Table 8 with PID, which occurred 1 month later.

Case 2: Age 37. Abdominal pain due to ovarian cys 7 months after fitting. Ovarian cystectomy was performed. Case 3: Age 33. 'Inflammation of the bowel' 5 days after fitting. Hospitalised. Septicaemia. Haemolytic streptococcus on blood culture. LNG IUS removed from uterus. History of Crohn's disease. The surgeon thought the LNG IUS may be the cause, the gynaecologist thought the Crohn's to be responsible.

Ovarian Cysts. In the present study 15 subjects developed an ovarian cyst before 12 months of use (2\% of 692 fittings). The LNG IUS was removed in four cases and in a fifth a ruptured ovarian cyst was removed.

\section{Positive views of the LNG IUS}

Many women are very happy with the reduction in the amount of bleeding following insertion of the LNG IUS. Positive comments are frequent, typically praising the system for its beneficial effects.

\section{Discussion}

Fitting experience shows that there appears to be a learning curve as most failed fittings were in the early months of enrolment. This has been reported in more detail elsewhere. ${ }^{13}$

The event rates are compared to other published studies in Table 1. All studies show very low pregnancy rates at 12 months. A Finnish study reported a pregnancy rate of 0.4 
Table 7 Medical reasons for removal by 12 months of use (considered to be associated with LNG IUS use) in 40 subjects, some having more than one complaint

\begin{tabular}{lc}
\hline Complaint & Number of complaint \\
\hline Oligo/amenorrhoea & 10 \\
Bloating & 8 \\
Premenstrual-type symptoms/PMT & 5 \\
Breast tenderness & 5 \\
Weight gain & 5 \\
Headaches & 4 \\
Nausea & 4 \\
Mood swings/depression & 3 \\
Tiredness/ 'felt unwell' & 3 \\
Acne & 3 \\
Dizziness & 3 \\
Palpitations & 2 \\
Ovarian cyst & 2 \\
Other $*$ & 11 \\
Total & 68
\end{tabular}

*Includes the following (one complaint each): migraine, hot sweats, oedema, discharge, dyspareunia, leg pain, backache, pelvic pain, breast lump, 'IUS felt low in cavity', septicaemia (see 'Deaths and other serious adverse events' under 'Serious adverse events').

after 5 years and a Population Council study ${ }^{6}$ reported a rate of 1.1 after 7 years. In the present study all three pregnancies resulted in spontaneous abortion. It has long been known that the spontaneous abortion rate is high when a pregnancy occurs in the presence of an IUD. ${ }^{14}$

The results of the present study are comparable to other studies except that the removal rates for bleeding and bleeding with pain are higher. The reason for this is not obvious. Perhaps the women have been less tolerant of side effects than in other studies.

The pattern of continuous loss over several months, or of disruption of bleeding patterns, experienced by the majority of the 60 cases of removal at or before 12 months for bleeding problems (Table 6) does not suggest a continuation of a previous pattern of menorrhagia/dysmenorrhoea. There is also a need to be aware of cultural drawbacks to abnormal bleeding. In this study a Muslim requested removal after 2 months of use because long periods of bleeding interfered with prayer. Nevertheless, this is a short-lived problem for many subjects, lasting for the first 3 months of LNG IUS use only.

Other studies have shown that when compared to the NovaT $^{\circledR}$, the rate of removal for bleeding problems with the LNG IUS in the first 12 months of use has been similar, but at 60 months has been much lower. For example a study in Scandinavia and Hungary ${ }^{12}$ found the net removal rates for bleeding problems with the LNG IUS compared to the Nova-T ${ }^{\circledR}$ at 12 months to be 5.8 and 5.7 , but at 60 months the rates were 10.9 and 16.2 respectively.
Oligomenorrhoea or amenorrhoea are more evident after the first 12 months (in this study oligomenorrhoea and amenorrhoea were not classified separately). Nevertheless 10 women had requested removal by 12 months for this reason (Table 7). Whether these changes are considered as positive or negative will vary between individuals. Many women in the present study commented favourably on the reduction of duration and quantity of menstrual bleeding. This reduction is such that the LNG IUS is finding an important place in the treatment of excessive uterine bleeding. ${ }^{15-17}$

There is therefore a need to counsel women about the early bleeding problems including the possibility of oligomenorrhoea or amenorrhoea. This is considered to be very important as it will help women to persevere so that they can enjoy the longer-term benefits. In this study all investigators carried out such counselling.

Can any of the side effects, other than bleeding disturbances, be due to levonorgestrel absorbed from the LNG IUS? Many symptoms have been associated with oral progestogens. For example in the present study there were complaints of breast pain, weight gain, headache, depression, premenstrual tension (Table 7).

The amount of levonorgestrel absorbed from an LNG IUS is very small. Studies show that there is a peak plasma level 2 hours after oral ingestion of a $20 \mu \mathrm{g}$ levonorgestre pill and a trough after 24 hours. ${ }^{18}$ Studies of the LNG IUS show a fairly constant plasma level of levonorgestrel similar to the trough levels after oral ingestion. ${ }^{19,20}$ Can a constant low level of levonorgestrel cause symptoms? Are some individuals more sensitive than others to low plasma levels of levonorgestrel? Double blind trials to give the answer are impossible.

Two studies ${ }^{21,22}$ showed that the incidence of some of these symptoms were actually greater before than during the administration of POPs. So perhaps some symptoms are not due to the levonorgestrel. Nevertheless these symptoms caused 40 women in the present study to have removal within the first 12 months.

The diagnosis of PID depended on the clinical judgement of the clinician involved as laparoscopy was not used. The six cases of PID could have been related to the LNG IUS. However, the frequency of PID has been shown in recent studies to be only minimally, if at all, related to the IUD. Of greater significance is exposure to sexually transmitted infection rather than the presence of an IUD. ${ }^{23}$ During the period of this study only some centres were carrying out screening and prophylactic measures for Chlamydia and other organisms. There appeared to be no increased risk of PID soon after fitting as has been reported elsewhere. ${ }^{24}$

In the present study 15 subjects developed an ovarian cyst before 12 months of use ( $2 \%$ of 692 fittings). In a study of 1821 fittings $^{25}$ there were three removals due to ovarian

Table 8 Pelvic inflammatory disease

\begin{tabular}{|c|c|c|c|c|c|}
\hline Age & Duration of IUD use & Severity & Investigation/Treatment & IUS removed & Result \\
\hline 29 & 4 months & severe & Hospitalisation, Augmentin ${ }^{\circledR}$ Metronidazole & yes & cured \\
\hline$* 29$ & 5 months & mild & $\begin{array}{l}\text { PID or UTI, Cephradine } \\
\text { Metronidazole, Erythromycin }\end{array}$ & yes & cured \\
\hline 41 & 4 months & severe & $\begin{array}{l}\text { Hospitalisation, Augmentin } \\
\text { Doxycycline }\end{array}$ & yes & cured \\
\hline \#34 & 6 months & moderate & Co-trimoxazole & yes & cured \\
\hline 40 & 1 year & severe & Hospitalisation, IV antibiotics & yes & cured \\
\hline 19 & 3 weeks & moderate & Hospitalisation, antibiotics & yes & cured \\
\hline
\end{tabular}

* Diagnosis doubtful. Could have been UTI.

\# Diagnosis doubtful. 
cysts up to 12 months of use. In another study functional ovarian cysts were found to be associated with the LNG IUS in six of 50 women. ${ }^{26}$

\section{Conclusion}

At 12 months of use the LNG IUS has been shown to have a very low pregnancy and a low expulsion rate. The rate of removal for a complaint of bleeding or bleeding with pain is high. Experience during the first year of use confirms the need for careful counselling before the device is fitted to prepare women for the changes of bleeding patterns to be encountered in the early months of use. Removals were also required for side effects which may be due to absorbed levonorgestrel. The reduction in duration and quantity of periods in many women were significant advantages.

\section{Acknowledgements}

The LNG IUSs were supplied by Leiras Oy. Statistical analysis by Ruth Preist, Hilary Hardwicke and Sarah Blacksell of the Institute of Population Studies, University of Exeter. Supervision by Dr John Tripp of the School of Postgraduate Medicine and Health Sciences, University of Exeter.

\section{Statements on funding and competing interests}

Funding. This project is sponsored by Leiras Oy and Schering Health Care Limited.

Competing interests. None.

References

Luukkainen T. The levonorgestrel-releasing IUD. Br J Fam Plann 1993; 19: 221-224 Andersson K, et al. Levonorgestrel-releasing and copper releasing (NovaT) IUDs during five years of use: A randomised comparative trial. Contraception 1994; 49: 56-72.

Indian Council of Medical Research Task Force on IUD.Randomised clinical trials with intrauterine devices levonotgestrel intra-uterine device (LNG), Cu T380Ag, Cu T220C, Cu T 200B): A 36-month study. Contraception 1989; 39: 37-52.
Luukkainen T, Allonen H, et al. Five years experience with levonorgestrel-releasing IUDs. Contraception 1986; 33: 139-148.

Sivin I, Stern J, et al. Prolonged intrauterine contraception: a seven year randomised study of the levonorgestrel 20 microgram/day, LNG-20 and the copper Ag IUDs. Contraception 1991; 44: $473-480$.

World Health Organisation (1987). Mechanism of action, safety and efficacy of intrauterin devices. Technical Report Series 753. Geneva: WHO.

Silverberg SG, Haukkamaa M, et al. Endometrial morphology during long-term use of

Sivin I, Stern J, and the International Committee for Contraception Research. Health during prolonged use of Levonorgestrel 20 micrograms/day and the Copper TCu $380 \mathrm{AG}$ intrauterine Chi I-c. The T Cu-380A (Ag), MLCu375, and Nova T IUDs and the IUD

and the IUD daily releasing 20 micrograms levonorgestrel - four pillars of IUD contraception for the nineties and beyond? Contraception 1993; 47: 325-347.

11 Sivin I, et al. Prolonged intrauterine contraception: a seven-year randomised study of the levonorgestrel 20 micrograms/day (LNG 20) and the Copper T380 Ag IUDs. Contraception 1979; 44(5): 473-480.

Tietze C, Lewitt S. Recommended procedures for the statistical evaluation of intrauterine contraception. Studies in Fam Plann 1973; 4(2): 35-42.

3 Tattersall J, Allison CJ, Blacksell S, in collaboration with the UK Family Planning Research Network. The Levonorgestrel Intrauterine System in routine use: experiences of insertion. Acta Obstet Gynecol Scand 1997; 76(Suppl. 167:4): P72.31, pp51.

4 Vessey M P, Johnson B, et al. Outcome of pregnancy in women using an intrauterine device. Lancet 1974; 1: 495-498.

Bounds W. Clinical experience with a levonorgestrel-releasing intrauterine contraceptive 193-194.

16 Sturridge F, Guillebaud J. A risk-benefit assessment of the levonorgestrel-releasing intrauterine system. Drug Safety 1996; 15(6): 430-440.

17 Royal College of Obstetricians and Gynaecologists. The Management of Menorrhagia in Secondary Care. Evidence-based Clinical Guidelines No5. July 1999.

18 Weiner E, Victor A, Johansson EDB. Plasma levels of d-norgestrel after oral administration. Contraception 1976; 14: 563-570.

Fotherby K. Levonorgestrel, clinical pharmacokinetics. Clin Pharmacokinet 1995; 28(3) $203-215$

Nilsson CG, Lahteenmaki PIA, et al. Sustained intrauterine release of levonorgestrel. Fer Steril 1986; 45: 805-807.

Korba VD, Paulson SR. Five years of fertility control with microdose Norgestrel: an update clinical review. J of Reprod Med 1974; 13: 71-75.

22 Scharff H J. Clinical experience with Microlut. Schering Med News 1972; 1: 2-8.

Bromham DR. Intrauterine contraceptive devices- a reappraisal. Br Med Bull 1993; 49. $100-123$

24 Farley TMH, Rosenberg MJ, Rowe P. Intrauterine devices and pelvic inflammatory disease: an international perspective. Lancet 1992; 339: 785-788.

25 Chi I-c. An evaluation of the levonorgestrel-releasing IUD: Its advantages and disadvantage when compared to the copper releasing IUDs. Contraception 1991; 44: 573-588.

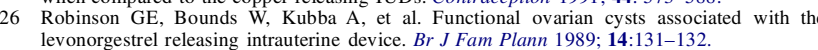

